


\section{Welcome Wolfgang Newerla to the JOSHA editorial board}

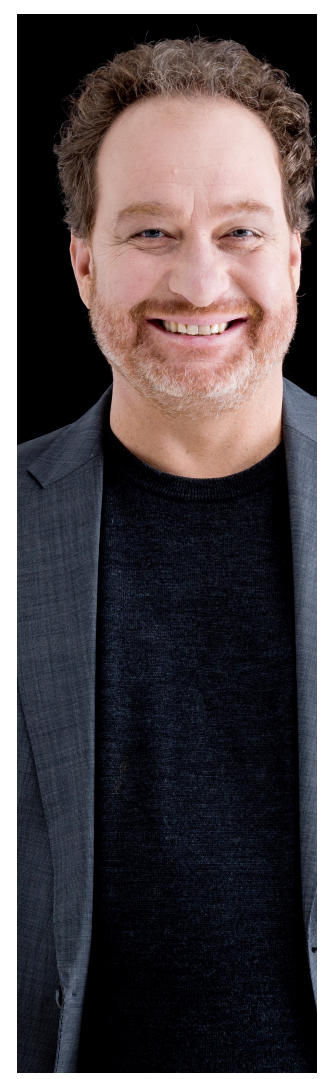

His great musical curiosity gave the baritone an early reputation as a singer with the most interesting repertoire of his field. After engagements in Ulm and Freiburg, he has been a guest at many important national and international opera houses such as the Theater an der Wien, the Vienna Volksoper, the Teatro Real in Madrid, the Opéra National de Lyon, the Staatsoper Berlin, the Deutsche Oper am Rhine, Staatsoper Hannover and festivals such as the Ruhrtriennale and the Schwetzingen Festival since1999 In June 2014 he debuted with great success at the Staatsoper Munich in a new production of Zimmermann's soldier (Kirill Petrenko / Director: Andreas Kriegenburg). Recently he was seen in Calixto Bieitos production of Janáček AUS EINEM TOTENHAUS at the Staatsstheater in Nuremberg. With Zimmermann's soldiers, he will make his debut at the Teatro Real in Madrid in the spring of 2018. Besides the opera, Wolfgang Newerla focuses on the concert sector. As a concert performer, he is very popular all over Europe - mainly in Spain, as well as in China, the USA, and Russia.

WOLFGANG NEWERLA ist seit vielen Jahren Gast an einer Reihe von wichtigen nationalen und internationalen Opernhäusern. Seine große musikalische Neugier verschaffte Wolfgang Newerla früh den Ruf, zu den Sängern mit dem interessantesten Repertoire seines Fachs zu gehören.

In den letzten Jahren debütierte er mit großem Erfolg an der Staatsoper München in einer Neuproduktion von Zimmermanns SOLDATEN als Mary (Kirill Petrenko/Regie: Andreas Kriegenburg). Diese Partie wird er 2018 auch am Teatro Real Madrid übernehmen.

2013 debütierte er in Georg Friedrich Haas' Uraufführung der Oper THOMAS mit der Partie des Matthias bei den Schwetzinger Schlossfestspielen. Im Jahr zuvor hatte Wolfgang Newerla einen großen persönlichen Erfolg in der Titelpartie von Carl Orffs PROMETHEUS in einer Neuproduktion der Ruhrtriennale. 2011 debütierte er an der Opéra de Lyon als Mann in Schönbergs VON HEUTE AUF MORGEN.

Er sang den Kommerzienrat in INTERMEZZO (Strauss) im Theater an der Wien, Tschekunoff und Don Juan in TOTENHAUS an der Staatsoper Hannover, Panthée/ Mercure in LES TROYENS an der Staatsoper Stuttgart und Hans Zenders CHIEF JOSEPH sowie den Dr. Vigelius in Schrekers DER FERNE KLANG an der Berliner Staatsoper Unter den Linden. An der Oper Leipzig sang er in Luca Lombardis DMITRI und Schönbergs VON HEUTE AUF MORGEN, an der Oper Nürnberg den Prospero in Luca Lombardis DER STURM, die Semperoper Dresden erlebte inn als Jupiter in Strauss' DIE LIEBE DER DANAE. 
Weitere Gastspiele führten ihn u.a. an die Volksoper Wien, das Teatro Real in Madrid, die Opéra National de Lyon, die Deutsche Oper Berlin und die Deutsche Oper am Rhein. 2011 debütierte er in der CARMINA BURANA bei den Münchener Opernfestspielen. Am Theater Freiburg und am Staatstheater Darmstadt gastierte er mit Gunther in DIE GÖTTERDÄMMERUNG in einer seiner ausgesuchten Wagner-Partien.

Ein grosses Interesse des Sängers gilt auch dem Barock-Repertoire. So war er u.a. an szenischen Produktionen von FILOSOFO NELLA CAMPAGNA (Galuppi), SERVA PADRONA (Pergolesi), SERVA SCALTRA (Hasse), PIMPINONE (Telemann), ALMIRA, SAUL und JEPHTA (Händel) beteiligt und musizierte dabei mit Orchestern wie dem Freiburger Barockorchester und der Akademie für Alte Musik Berlin.

Wolfgang Newerla ist als Konzertsänger sehr gefragt. Konzerte gibt er - mit Schwerpunkt Spanien - in ganz Europa, darüber hinaus auch in China, den USA und Russland.

Der Künstler arbeitete mit Dirigenten wie Zubin Mehta, Ivor Bolton, Kirill Petrenko, Michael Gielen, Julia Jones, Phillippe Jordan, Thomas Hengelbrock, Heinz Wallberg, Axel Kober, Hermann Max, Sylvain Cambreling, Manfred Honeck, Christopher Hogwood und Roy Goodman sowie mit Regisseuren wie Peter Mussbach, Christoph Loy, Barrie Kosky, Gerd Heinz, Michael Simon und Anthony Pilavachi.

Wolfgang Newerla war Preisträger des Belvedere Wettbewerbs Wien. Seine musikalische Ausbildung erhielt er in München, Detmold und Hamburg. Erste Festengagements führten inn nach Ulm und Freiburg.

Für seine Interpretation des Cortez in Wolfgang Rihms DIE EROBERUNG VON MEXICO wurde er von der Zeitschrift Opernwelt mit einer Nominierung zum Sänger des Jahres bedacht. 